\title{
High-speed time-reversed ultrasonically encoded (TRUE) optical focusing inside dynamic scattering media at $793 \mathrm{~nm}$
}

Yan Liu, Puxiang Lai, Cheng Ma, Xiao Xu, Yuta Suzuki, et al.

Yan Liu, Puxiang Lai, Cheng Ma, Xiao Xu, Yuta Suzuki, Alexander A. Grabar, Lihong V. Wang, "High-speed time-reversed ultrasonically encoded (TRUE) optical focusing inside dynamic scattering media at $793 \mathrm{~nm}$," Proc. SPIE 8943, Photons Plus Ultrasound: Imaging and Sensing 2014, 894339 (3 March 2014); doi: 10.1117/12.2039815

SPIE. Event: SPIE BiOS, 2014, San Francisco, California, United States 


\title{
High-Speed Time-Reversed Ultrasonically Encoded (TRUE) Optical Focusing inside Dynamic Scattering Media at 793 nm
}

\author{
Yan Liu ${ }^{1, \dagger}$, Puxiang Lai ${ }^{1, \dagger}$, Cheng Ma ${ }^{1}$, Xiao Xu ${ }^{1}$, Yuta Suzuki ${ }^{1}$, Alexander A. Grabar ${ }^{2}$ and Lihong \\ V. Wang ${ }^{1, *}$ \\ ${ }^{1}$ Optical Imaging Laboratory, Department of Biomedical Engineering, Washington University, \\ Saint Louis, Missouri 63130, USA \\ 2 Institute of Solid State Physics and Chemistry, Uzhgorod National University, 88000 Uzhgorod, \\ Ukraine \\ ${ }^{\dagger}$ Equal contribution *Corresponding author: 1hwang@wustl.edu
}

\begin{abstract}
Time-reversed ultrasonically encoded (TRUE) optical focusing is an emerging technique that focuses light deep into scattering media by phase-conjugating ultrasonically encoded diffuse light. In previous work, the speed of TRUE focusing was limited to no faster than $1 \mathrm{~Hz}$ by the response time of the photorefractive phase conjugate mirror, or the data acquisition and streaming speed of the digital camera; photorefractive-crystal-based TRUE focusing was also limited to the visible spectral range. These time-consuming schemes prevent this technique from being applied in vivo, since living biological tissue has a speckle decorrelation time on the order of a millisecond. In this work, using a Tedoped $\mathrm{Sn}_{2} \mathrm{P}_{2} \mathrm{~S}_{6}$ photorefractive crystal at a near-infrared wavelength of $793 \mathrm{~nm}$, we achieved TRUE focusing inside dynamic scattering media having a speckle decorrelation time as short as $7.7 \mathrm{~ms}$. As the achieved speed approaches the tissue decorrelation rate, this work is an important step forward toward in vivo applications of TRUE focusing in deep tissue imaging, photodynamic therapy, and optical manipulation.
\end{abstract}

Keywords: Time-Reversed Ultrasonically Encoded (TRUE) optical focusing, optical phase conjugation, optical time reversal, light scattering, turbid media, optical focusing.

\section{INTRODUCTION}

Light focusing plays a central role in biomedical imaging, optical manipulation and therapy. However, in scattering media, direct light focusing deeper than around 10 scattering mean free path (typically $\sim 1 \mathrm{~mm}$ in biological tissue) becomes infeasible due to light scattering ${ }^{1-3}$. Overcoming this optical diffusion limit has been extensively studied within the past decades. For example, wavefront shaping ${ }^{4,5}$ has demonstrated that light can be focused through scattering media by compensating the scattering-induced phase distortions along the optical paths. Obtaining the best phase correction, however, is usually a time-consuming process (seconds to hours) limited by the refreshing speed of the spatial light modulators and the iterative nature of optimization. A solution to this speed limitation, termed timereversed ultrasonically encoded (TRUE) optical focusing ${ }^{6-11}$, uses either an analog (photorefractive material) or a digital (a digital camera plus a spatial light modulator) phase conjugate mirror to selectively record and time-reverse the ultrasound-modulated light emitted from the virtual guide-star formed by acoustic focus inside the tissue. TRUE focusing is inherently faster than wavefront shaping since it measures the desired phase map directly, avoiding the timeconsuming exhaustive search for the optimum wavefront in wavefront shaping. However, the speed of TRUE focusing was limited to no faster than $1 \mathrm{~Hz}$ by the response time of the photorefractive phase conjugate mirror (for the analog approach $^{6,12}$ ), or the data acquisition and streaming speed of the digital camera (for the digital approach ${ }^{8,9}$ ). Moreover, photorefractive-crystal-based TRUE focusing was also limited to the visible spectral range, limiting its capability to focus even deeper. So far, there has been no report of studies on optical focusing deep inside dynamic scattering media or living biological tissue, mainly because the time needed to achieve focusing in previous work was much longer than the speckle decorrelation time of the scattering media, which is on the order of $1 \mathrm{~ms}$ for living tissue as determined by motions such as blood flow, breathing and heartbeat. In this work, we present our latest explorations, using a fast Te

Photons Plus Ultrasound: Imaging and Sensing 2014, edited by Alexander A. Oraevsky, Lihong V. Wang, Proc. of SPIE Vol. 8943, 894339 - (C) 2014 SPIE · CCC code: 1605-7422/14/\$18 - doi: 10.1117/12.2039815 
doped $\mathrm{Sn}_{2} \mathrm{P}_{2} \mathrm{~S}_{6}$ photorefractive crystal (provided by Prof. Alexander A. Grabar) as a phase conjugate mirror, to focus light inside dynamic scattering media with a speckle decorrelation time as short as $7.7 \mathrm{~ms}$.

\section{METHODS}

\subsection{Experimental set-up for TRUE optical focusing inside dynamic scattering media}

Fig. 1 shows a diagram of the main parts of the set-up. The detailed layout is similar to that in Ref. 13. A continuouswave Ti:Sapphire laser (MBR110, Coherent) operating at $793 \mathrm{~nm}$ was used as the light source. A reference beam R and ultrasonically tagged signal light $\mathrm{S}$ wrote a holograph in the $\mathrm{Sn}_{2} \mathrm{P}_{2} \mathrm{~S}_{6}$ :Te photorefractive crystal (PRC) for $10 \mathrm{~ms}$. Then, the writing beams were blocked by a mechanical shutter and the holograph was read by another reference beam $\mathrm{R}^{*}$ (conjugate of R) for $2 \mathrm{~ms}$, which produces a phase-conjugated signal light $\mathrm{S} *$ that converges back to the acoustic focus and was detected by a photodiode (PDA-36A, $70 \mathrm{~dB}$ gain, Thorlabs). The ultrasound was on only during the writing of the holograph. The $\mathrm{Sn}_{2} \mathrm{P}_{2} \mathrm{~S}_{6}$ :Te crystal was a $6 \times 6 \times 6 \mathrm{~mm}^{3}$ cube and the intensity of the writing beams on the crystal was $1.1 \mathrm{~W} / \mathrm{cm}^{2}$.

An absorptive target (made by adding black ink in gelatin) was sandwiched between two scattering media - a diffuser (DG10-120, Thorlabs), and an intralipid-gelatin phantom with a thickness of $2 \mathrm{~mm}$ (equivalent to 7 mean free paths at $793 \mathrm{~nm}$ ). The intralipid-gelatin phantom, mounted on a motorized linear stage, translates along the $\mathrm{x}$-direction at a controllable speed during the recording and reading of the holograph. The movement of the intralipid-gelatin phantom causes the interference pattern formed by $\mathrm{S}$ and $\mathrm{R}$ beams on the PRC to decorrelate, and we can control the speckle decorrelation time by translating the phantom at different speeds.

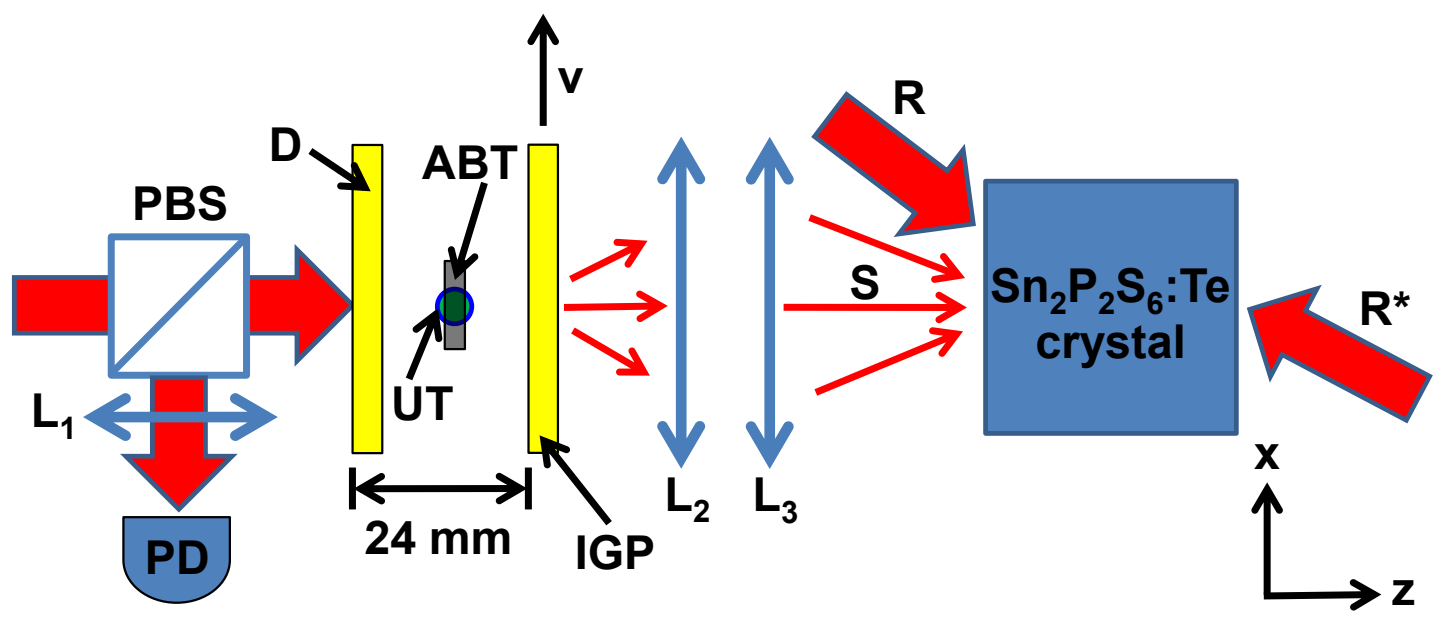

Figure 1. Diagram for the experiment on TRUE optical focusing inside dynamic scattering media. An absorptive target was sandwiched between a diffuser and an intralipid-gelatin phantom which moves along $\mathrm{x}$ direction at speed v. PBS, polarizing beam splitter; PD, photodiode; Li, ith lens; PD, photodiode; D, diffuser; ABT: absorptive bar target; UT, ultrasonic transducer; IGP, intralipid-gelatin phantom; S, signal light; R, reference beam; $R^{*}$, conjugated reference beam.

\subsection{Characterizing the relationship between speckle decorrelation time and phantom moving speed}

We characterized the relationship between speckle decorrelation time and phantom moving speed using the set-up shown in Fig. 2. The distance between the rear surface of the phantom and the focal plane of the objective was $22 \mathrm{~mm}$, which was limited by the dimensions of the phantom mount. At each phantom moving speed $\mathrm{v}$, we recorded a sequence of speckle patterns using a finite conjugate microscope objective $[10 \times$, numerical aperture $(\mathrm{NA})=0.1$, tube length $=$ $160 \mathrm{~mm}$, Leica] and a CMOS camera (208 frame per second, 320×108 pixels, Firefly MV, Point grey). We calculated the correlation coefficient between the first and each of the ensuing frames of the recorded speckle patterns. By fitting 
the curve of speckle correlation coefficient versus time using a Gaussian function, we obtained the speckle decorrelation time $T_{d}$, defined here as the time when the speckle correlation coefficient decreases to 0.1 , for this speed.

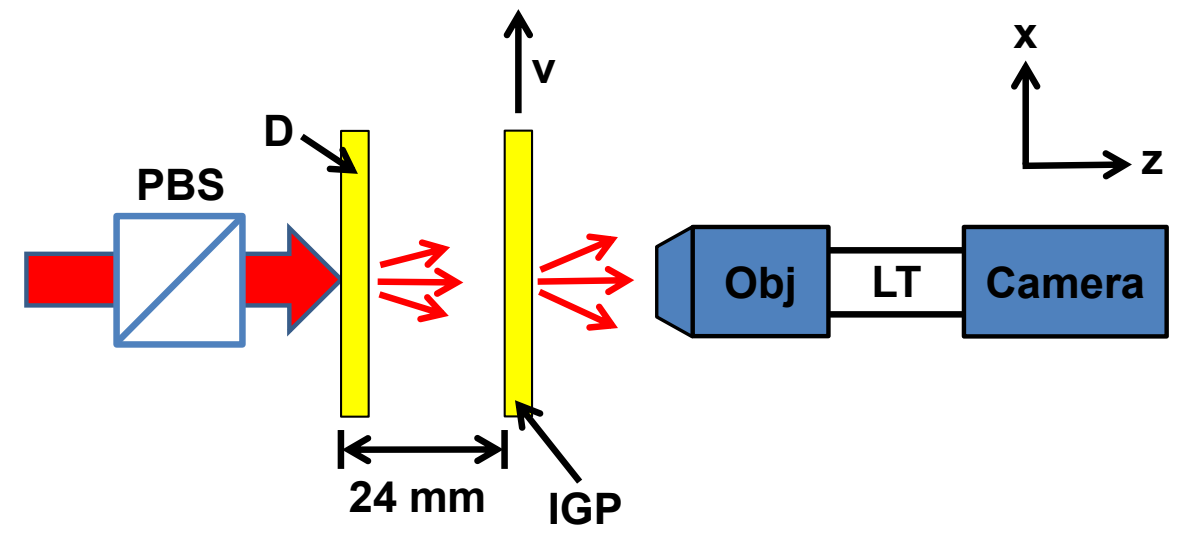

Figure 2. Experimental set-up to measure the speckle decorrelation time. PBS, polarizing beam splitter; D, diffuser; IGP, intralipid-gelatin phantom; Obj, microscope objective; LT, lens tube.

\section{RESULTS}

A sequence of speckle patterns when the intralipid-gelatin phantom was moving at a speed of $0.55 \mathrm{~mm} / \mathrm{s}$ was shown in Video 1 . We can see there are translations and transformations of the speckle patterns.

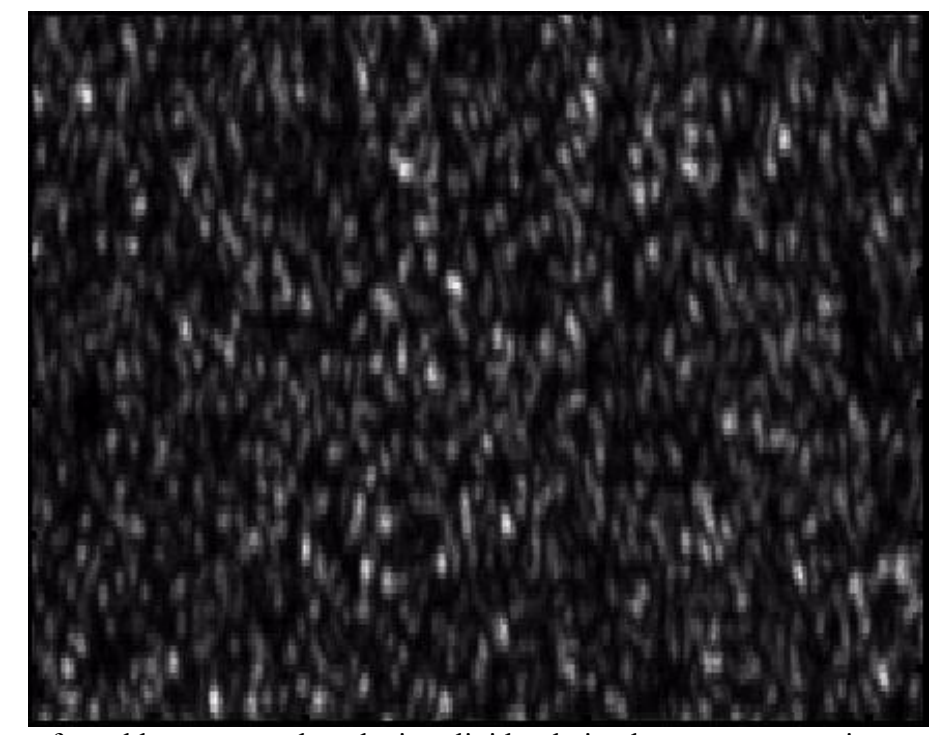

Video 1. A sequence of speckle patterns when the intralipid-gelatin phantom was moving at a speed of $0.55 \mathrm{~mm} /$ s. The video has a playback frame rate of $15 \mathrm{fps}$. http://dx.doi.org/0.1117/12.2039815.1

The speckle correlation coefficient vs. time when the phantom moving speed is $0.055 \mathrm{~mm} / \mathrm{sis}$ shown in Fig. 3 , from which we can obtain $T_{d}=72 \mathrm{~ms}$ for this speed. The value of $T_{d}$ as a function of phantom moving speed $\mathrm{v}$ is shown in Fig. 4. By fitting the experimental data with $T_{d}=a / v$, we obtain $a=4.23 \pm 0.12 \mu \mathrm{m}\left(R^{2}=0.9987\right.$ for the fitting). Using $\mathrm{T}_{\mathrm{d}}=4.23 \mu \mathrm{m} / \mathrm{v}$, we obtained $\mathrm{T}_{\mathrm{d}}=7.7 \mathrm{~ms}$ when $\mathrm{v}=0.55 \mathrm{~mm} / \mathrm{s}$ (at this speed, it is impossible to measure the speckle decorrelation time accurately by camera since the frame rate is $208 \mathrm{fps}$ ). 


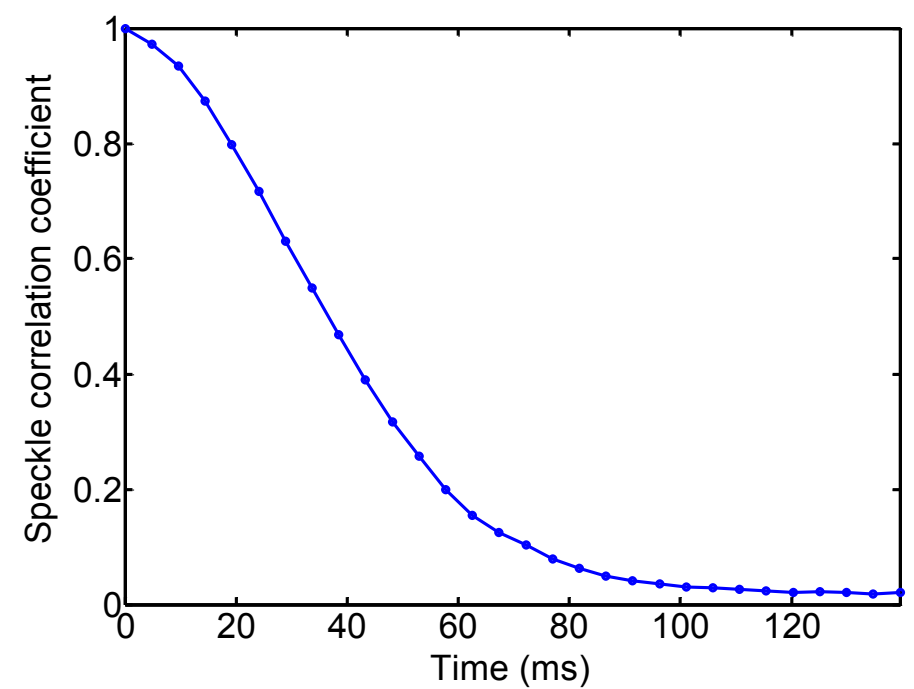

Fig. 3 The speckle correlation coefficient vs. time when the phantom moving speed was $0.055 \mathrm{~mm} / \mathrm{s}$.

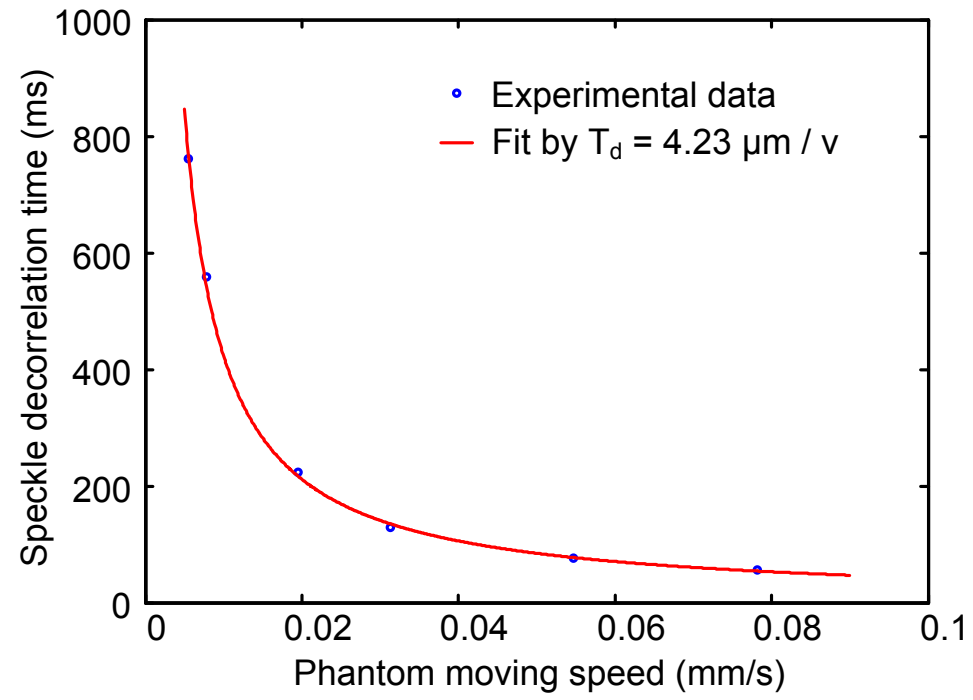

Fig. 4 The relationship between speckle decorrelation time and phantom moving speed.

When the phantom was moving at a speed of $\mathrm{v}=0.55 \mathrm{~mm} / \mathrm{s}$, the signals detected by the photodiode, when the ultrasound was on and off during writing the holograph, are shown in Fig.5. It can be seen that the maximum signal amplitude was always higher when the ultrasound was on than that when the ultrasound was off, which suggests that we could detect phase-conjugated light when $T_{d}=7.7 \mathrm{~ms}$. We did not observe the phase conjugated light when we shifted the ultrasound frequency by $100 \mathrm{kHz}$.

To acquire an image of the absorptive target with a width of $3.6 \mathrm{~mm}$, we scanned the gelatin phantom containing the target along the x-direction. At each scanning position, 10 TRUE procedures were performed and the signals were averaged. The one dimensional images of the target are shown in Fig. 6, for two cases - when the intralipid-gelatin phantom was static $\left(T_{d}>300 \mathrm{~ms}\right)$ and when it moved at a speed of $0.55 \mathrm{~mm} / \mathrm{s}\left(T_{d}=7.7 \mathrm{~ms}\right)$. It can be seen that the image acquired when $T_{d}=7.7 \mathrm{~ms}$ has a similar quality as the one acquired when $T_{d}>300 \mathrm{~s}$, demonstrating that our system is fast enough to overcome the speckle decorrelation caused by the movement of the scattering medium. The resolution of the image is about $1 \mathrm{~mm}$, which is close to the theoretical value of $0.87 \mathrm{~mm}$ determined by the ultrasonic transducer $(3.5 \mathrm{MHz}, \mathrm{NA}=0.25)$. 


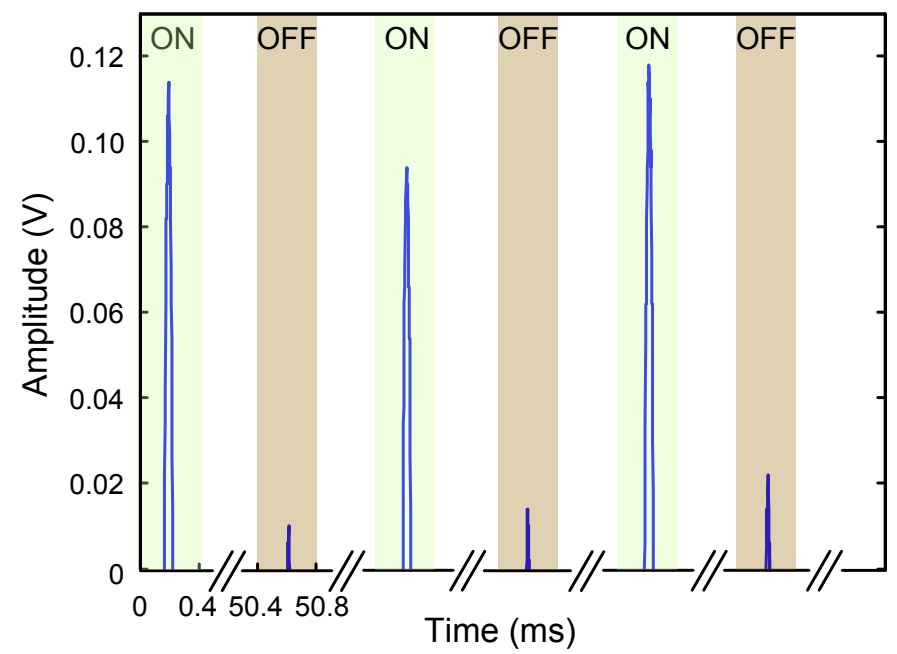

Fig. 5 The signal detected by the photodiode when the ultrasound was on and off during the writing of the holograph.

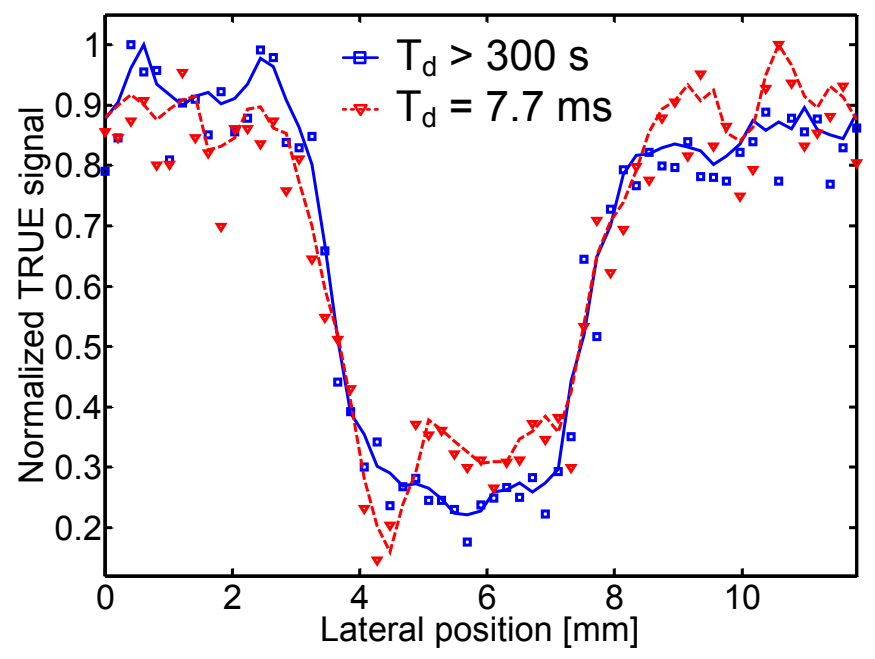

Fig. 6 One dimensional images of the absorptive target, when the intralipid-gelatin phantom was static $\left(\mathrm{T}_{\mathrm{d}}>300 \mathrm{~ms}\right)$ and when the phantom was moving at a speed of $0.55 \mathrm{~mm} / \mathrm{s}\left(\mathrm{T}_{\mathrm{d}}=7.7 \mathrm{~ms}\right)$. Solid and dashed lines represent smoothed data computed by a 4-point moving average of the raw data. The step size of scanning is $0.2 \mathrm{~mm}$.

\section{CONCLUSIONS}

Using a Te-doped $\mathrm{Sn}_{2} \mathrm{P}_{2} \mathrm{~S}_{6}$ photorefractive crystal at a near-infrared wavelength, we achieved high-speed TRUE focusing inside moving scattering media with a speckle decorrelation time as short as $7.7 \mathrm{~ms}$. As the demonstrated speed approaches the tissue decorrelation rate, this work is an important step forward toward in vivo applications of TRUE focusing in deep tissue imaging, photodynamic therapy, and optical manipulation. 


\section{ACKNOWLEDGEMENT}

This work was sponsored in part by National Institute of Health grants DP1 EB016986 (NIH Director's Pioneer Award) and R01 CA186567 (NIH Director's Transformative Research Award) as well as National Academies Keck Futures Initiative grant IS 13. L. W. has a financial interest in Microphotoacoustics, Inc. and Endra, Inc., which, however, did not support this work.

\section{REFERENCES}

[1] Y. Liu, C. Zhang and L. V. Wang, "Effects of light scattering on optical-resolution photoacoustic microscopy," $J$ Biomed Opt 17(12), 126014 (2012).

[2] L. V. Wang and G. Liang, "Absorption distribution of an optical beam focused into a turbid medium," Appl Optics 38(22), 4951-4958 (1999).

[3] V. Ntziachristos, "Going deeper than microscopy: the optical imaging frontier in biology," Nat Methods 7(8), 603614 (2010).

[4] I. M. Vellekoop and A. P. Mosk, "Focusing coherent light through opaque strongly scattering media," Opt. Lett. 32(16), 2309-2311 (2007).

[5] A. P. Mosk, A. Lagendijk, G. Lerosey and M. Fink, "Controlling waves in space and time for imaging and focusing in complex media," Nat Photon 6(5), 283-292 (2012).

[6] X. Xu, H. Liu and L. V. Wang, "Time-reversed ultrasonically encoded optical focusing into scattering media," Nat Photon 5(3), 154-157 (2011).

[7] P. Lai, X. Xu, H. Liu and L. V. Wang, "Time-reversed ultrasonically encoded (TRUE) optical focusing in biological tissue," J Biomed Opt 17(3), 030506 (2012).

[8] Y. M. Wang, B. Judkewitz, C. A. DiMarzio and C. Yang, "Deep-tissue focal fluorescence imaging with digitally time-reversed ultrasound-encoded light," Nat Commun 3(928 (2012).

[9] K. Si, R. Fiolka and M. Cui, "Fluorescence imaging beyond the ballistic regime by ultrasound-pulse-guided digital phase conjugation," Nat Photon 6(10), 657-661 (2012).

[10] P. Lai, Y. Suzuki, X. Xu and L. V. Wang, "Focused fluorescence excitation with time-reversed ultrasonically encoded light and imaging in thick scattering media," Laser Physics Letters 10(7), 075604 (2013).

[11] Y. Suzuki, X. Xu, P. Lai and L. V. Wang, "Energy enhancement in time-reversed ultrasonically encoded optical focusing using a photorefractive polymer," J Biomed Opt 17(8), 080507 (2012).

[12] P. Lai, X. Xu, H. Liu, Y. Suzuki and L. V. Wang, "Reflection-mode time-reversed ultrasonically encoded optical focusing into turbid media," J Biomed Opt 16(8), 080505 (2011).

[13] H. Liu, X. Xu, P. Lai and L. V. Wang, "Time-reversed ultrasonically encoded optical focusing into tissuemimicking media with thickness up to 70 mean free paths," J Biomed Opt 16(8), 086009 (2011). 